\title{
《环境昆虫学报》第四届编辑委员会 JOURNAL OF ENVIRONMENTAL ENTOMOLOGY EDITORIAL BOARD
}

主编 Editor-in-chief: 庞 虹 PANG Hong

荣誉主编 Honorary editor-in-chief: 李丽英 LI Li-Ying 庞 义PANG Yi

副主编 Associate editors-in-chief:

陈学新 CHEN Xue-Xin

陆永跃 LU Yong-Yue

曾金年 ZENG Xin-Nian

委员 Members (按姓氏拼音排序):

操海群 CAO Hai-Qun

陈法军 CHEN Fa-Jun

褚 栋 CHU Dong

冯启理 FENG Qi-Li

桂富荣 GUI Fu-Rong

姜立云 JIANG Li-Yun

李 胜 LI Sheng

李志强 LI Zhi-Qiang

刘怀 LIU Huai

任国栋 REN Guo-Dong

王 敦 WANG Dun

王满困 WANG Man-Qun

温俊宝 WEN Jun-Bao

谢 强 XIE Qiang

杨益众 YANG Yi-Zhong

藏连生 ZANG Lian-Sheng

章玉苹 ZHANG Yu-Ping
韩日畴 HAN Ri-Chou

王桂荣 WANG Gui-Rong

潘志萍 (常务) PAN Zhi-Ping
陈 兵 CHEN Bing

陈祥盛 CHEN Xiang-Sheng

DE CLERCQ Patrick

冯 夏 FENG Xia

侯有明 HOU You-Ming

李朝飞 LI Chao-Fei

李后魂 LI Hou-Hun

梁 沛 LIANG Pei

罗开珺 LUO Kai-Jun

沈 杰 SHEN Jie

王 建 WANG Su

王四宝 WANG Si-Bao

吴青君 WU Qing-Jun

许永玉 XU Yong-Yu

虞国跃 YU Guo-Yue

张爱兵 ZHANG Ai-Bing

钟国华 ZHONG Guo-Hua
洪晓月 HONG Xiao-Yue

张文庆 ZHANG Wen-Qing

陈大福 CHEN Da-Fu

陈晓光 CHEN Xiao-Guang

EHLERS Ralf-Udo

符悦冠 FU Yue-Guan

黄焕华 HUANG Huan-Hua

李飞 LI Fei

李有志 LI You-Zhi

刘桂林 LIU Gui-Lin

吕要斌 LV Yao-Bin

SLIPINSKI Adam

王建国 WANG Jian-Guo

王小艺 WANG Xiao-Yi

武予清 WU Yu-Qing

杨 朗 YANG Lang

余小强 YU Xiao-Qiang

张春田 ZHANG Chun-Tian

周忠实 ZHOU Zhong-Shi

本期执行主编: 曾釒年 组稿: 陈 兵 责任编辑: 潘志萍 翟 欣

\section{环境昆虫学报}

HUANJING KUNCHONG XUEBAO

(双月刊, 2008 年前刊名为《昆虫天敌》)

第 42 卷 第 3 期 2020 年 5 月 5 日

主管单位: 广东省科学技术协会

主办单位: 广东省昆虫学会

中国昆虫学会

\section{出版单位: 科紫出殿社}

主编: 庞虹

编辑: 《环境昆虫学报》编辑部

地 址：广州市新港西路 105 号, 510260

电话: 020-84456131

电子邮件：insect1979@163.com

印刷装订：广州一龙印刷有限公司

国内发行: 广东省报刊发行局

国内订购：全国各地邮政局

海外总发行：中国国际图书贸易总公司

广告经营许可证: 000012070012
JOURNAL OF ENVIRONMENTAL ENTOMOLOGY

(Bimonthly, previous name Natural Enemies of Insects ) Vol. 42 No. 3 May 5, 2020

Managed by Guangdong Provincial Association for Science \& Technology

Sponsored by Guangdong Entomological Society \& Entomological Society of China

Published by Science Press

Editor-in-Chief: PANG Hong

Edited by Editorial Board of Journal of Environmental Entomology

Address: 105W Xingang Road, Guangzhou 510260, China.

Tel: $+86-20-84456131$

E-mails : insect1979@163. com

Printed by Printing Factory of Guangzhou Yilong Printing Co., Ltd.

Distributed by Guangdong Bureau for Distribution of Newspapers and Journals

Domestic subscription All Local Post Offices in China

Overseas subscription China International Book Trading Corporation 